\title{
Bioinformation
}

\author{
www.bioinformation.net
}

Web Server

\section{GeneVenn - A web application for comparing gene lists using Venn diagrams}

\author{
Mehdi Pirooznia, Vijayaraj Nagarajan and Youping Deng* \\ Department of Biological Sciences, University of Southern Mississippi, Hattiesburg, Mississippi - 39406, USA; \\ Youping Deng* - Email - youping.deng@usm.edu; Phone: +1601 266 6678; Fax: +1601 266 5797; \\ * Corresponding author \\ received June 02, 2006; accepted January 30, 2007; published online April 10, 2007
}

\begin{abstract}
:
Numerous methods are available to compare results of multiple microarray studies. One of the simplest but most effective of these procedures is to examine the overlap of resulting gene lists in a Venn diagram. Venn diagrams are graphical ways of representing interactions among sets to display information that can be read easily. Here we propose a simple but effective web application creating Venn diagrams from two or three gene lists. Each gene in the group list has link to the related information in NCBI's Entrez Nucleotide database.
\end{abstract}

Key Words: microarray: Venn diagrams; comparison; gene list; graphics

Availability: GeneVenn is available for free at http://mcbc.usm.edu/genevenn/

Background:

Microarray technologies are increasingly being used in biological and medical sciences for high throughput analysis of genetic information at genome levels. These types of analyses generate vast amounts of data, often in the form of large lists of genes differentially expressed between different sample sets. It leaves the researchers with the task of identifying the functional relevance of the observed expression changes. There are a number of methods to compare results from multiple microarray experiments. [2] Methods can be used to validate results from similar experiments performed under different conditions. One of the simplest but most effective of these procedures is to examine the overlap of resulting gene lists in a Venn diagram.

Venn diagram was introduced by John Venn in 1881. [5] It is the illustration based on set theory concepts. It is used to compare and contrast two or more than two items in terms of the relationship between them. The relationship could be the intersection, union, complement, etc.

Venn diagrams are used to show the mathematical or logical relationships between different groups of sets. A Venn diagram shows all the logical relations between the sets. Venn diagrams can provide much more information to the researcher. Venn diagrams are very useful to show graphically how the objects in the sets are similar, different or apparently distinct to each other.

Simple Venn diagrams are already being used in microarray data analysis software packages such as commercial GeneSpring $^{\circledR}$ and SilicoCyte ${ }^{\circledR}$ or open source R-package limma to visualize intersections of up to three different lists of genes. In the present paper, we propose a web application creating a Venn diagrams from two or three gene lists. It is been graphically designed and publicly available at http://mcbc.usm.edu/genevenn/.

\section{Implementation}

The design of GeneVenn follows that of a two tiers web application. The UML class diagram including application's class variables and methods is illustrated in Figure 1.

The user-friendly web interface has been developed by using the PHP language, DHTML, and JavaScript. The application is currently running under an Apache web server version 2.0 based on Linux Suse 10 OS.

\section{Content}

GeneVenn is relatively small but, nonetheless, effectively complete. In this, the initial welcome page has three text lists. A user is able to enter the gene names to these text areas as well as uploading gene lists files to the server and process them automatically. If the user enters data in the text box and uploads a gene file at the same list, data will be merged and considered as a single list. It is also possible to select the number of diagrams (two or three), set up a name for each diagram, and a title for the result. Any white space such as tab, space, line break or comma is accepted as a gene name delimiter.

The result page processes the lists and creates a Venn diagram. Each area on the diagram has a hyperlink which shows the related gene list and each gene name has linked to the related information in NCBI's Entrez Nucleotide database. Here, user is able to modify every element of the generated diagram, including font, color and name of the diagram. The Figure 2 illustrates the result page. 


\section{Bioinformation}

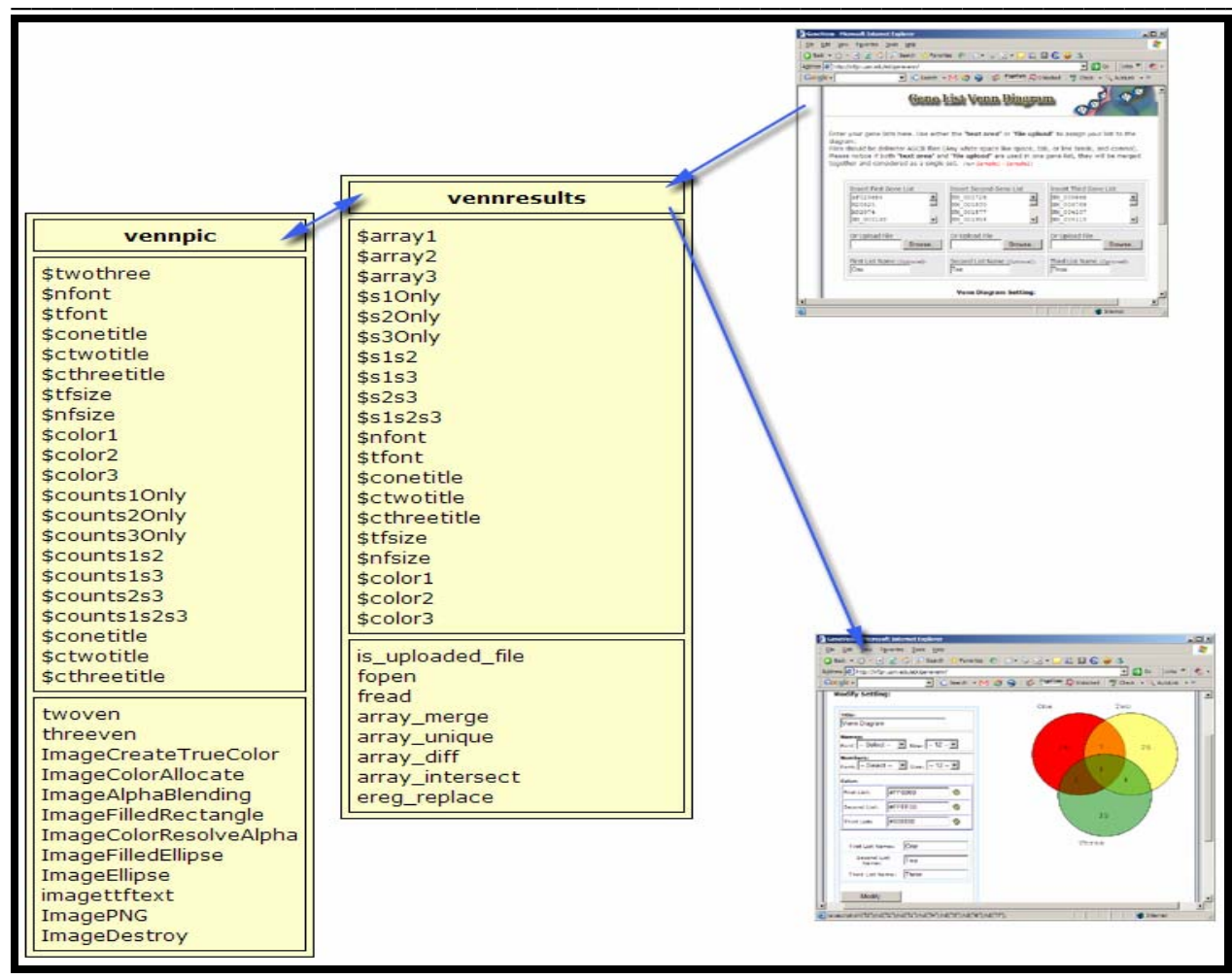

Figure1: GeneVenn UMLDiagram

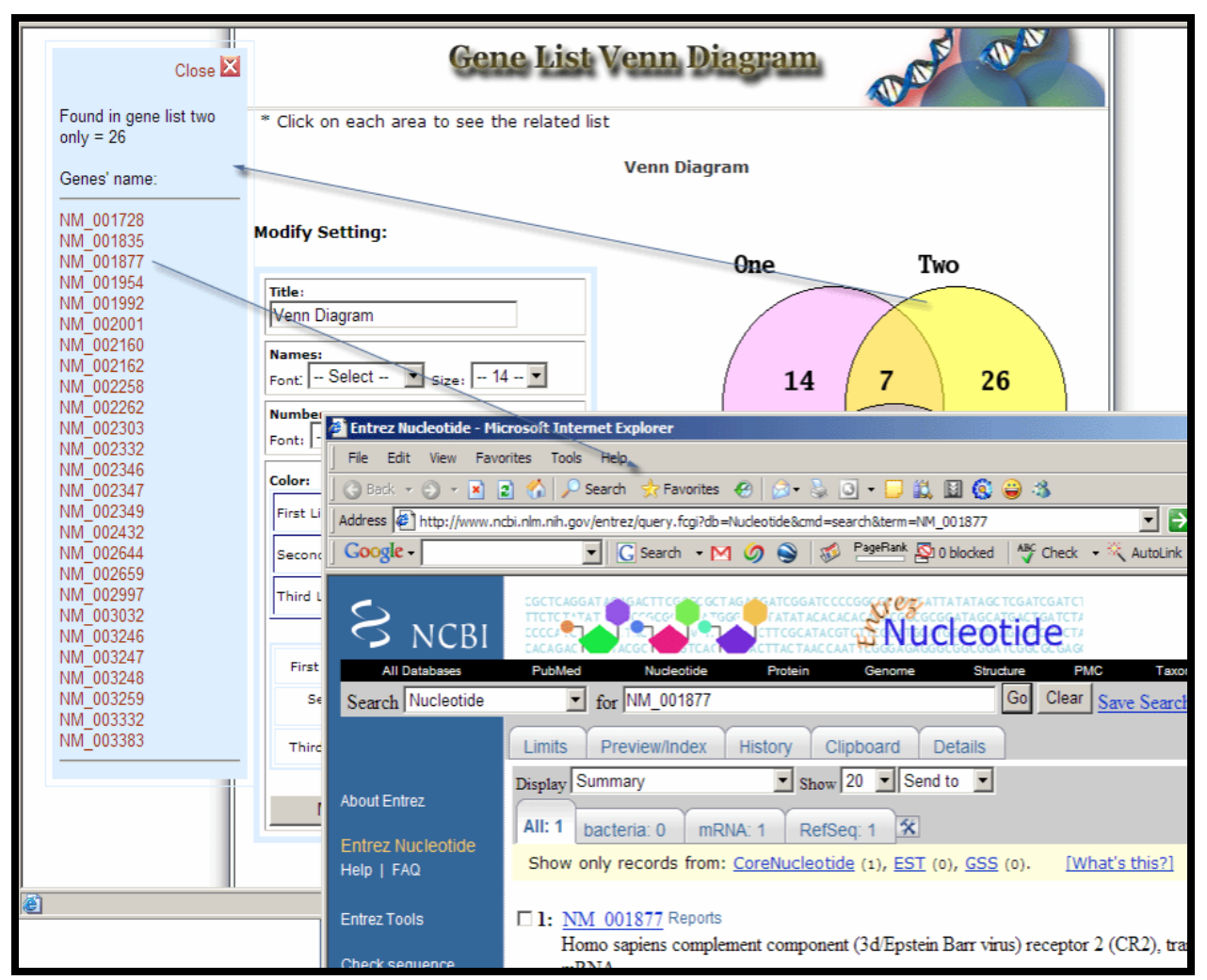

Figure2: The GeneVenn result page 


\section{Bioinformation}

open access

\section{www.bioinformation.net}

\section{Web Server}

System requirements

Operating systems: platform independent

Programming language: PHP, DHTML and JavaScript

License: GNU GPL

Any restrictions to use by non-academics: none

Acknowledgement:

This work was supported by Dean's Research Initiative award of the University of Southern Mississippi to Youping Deng and the Mississippi Functional Genomics Network (DHHS/NIH/NCRR Grant\# 2P20RR016476-04).

\section{References:}

[01] H. Cundy \& A. Rollett, Mathematical Models, 255:256 (1989)

[02] H. A. Kestler, et al., Bioinformatics, 21:1595 (2005) [PMID: 15572472]

[03] C. S. Ogilvy, Amer. Math., 62:584 (1955)

[04] F. Ruskey, Electronic J. Combinatorics Dynamical Survey, DS5 (1997)

[05] J. Venn, Philosophical Magazine and Journal of Science, 1:18 (1880)

Edited by Susmita Datta

Citation: Pirooznia et al., Bioinformation 1(10): 420-422 (2007)

License statement: This is an open-access article, which permits unrestricted use, distribution, and reproduction in any medium, for non-commercial purposes, provided the original author and source are credited. 